\title{
Neuromuscular environment and stability of transverse maxillary expansion
}

\author{
M.TsangTung ${ }^{1}$, M. Makaremi², F. de Brondeau ${ }^{3}$ \\ 1 Specialist in dentofacial orthodontics, University of Bordeaux \\ 2 Qualified specialist in dentofacial orthopedics, private practice, Anthropology Researcher \\ 3 Qualified specialist in dentofacial orthopedics, private practice, University lecturer
}

\section{SUMMARY}

Transverse maxillary insufficiency is one of the many dysmorphoses present in modern man. Since masticatory function is more affected by changes in diet and lifestyle, the stimulation of growth of the alveolar processes is weaker. The constriction of the maxilla is often the cause of dentomaxillary dysharmonia which requires space to be found on the arch. Expansion or extraction? This question has always stirred up our profession through the centuries.

However, the main part of these treatments is to obtain a stable occlusion in the long term and for this, management of the functional equilibrium is paramount. The neuromuscular environment participating in the morphogenesis of the dental arches, it is essential to take into account all these factors to potentiate the success of our treatments and to promote a harmonious.

\section{KEYWORDS}

Neuromuscular, maxillary expansion, masticatory strain, retention and stability, relapse

\section{INTRODUCTION}

Managing the stability of the transverse dimension after maxillary expansion is a serious problem because of the high rate of relapse, and studies are necessary for improving management and ensuring its sustainability. The correction of dysfunction is essential to avoid any possible relapses in the transverse dimension.

\section{Evolution of orthodontic philosophy}

The therapeutic indication for avulsions as a treatment option has varied since
Fauchard ${ }^{26}$, pioneer of modern dentistry, who treated dental irregularities by expanding the arches. There are two opposing extreme approaches: systematic preservation of all the teeth by expansion or avulsion to comply with cephalometric and aesthetic standards.

Relying on the laws set by Roux and Wolff affirming the predominant influence of functional factors on morphogenesis, Angle attributed the role of functional stimulation of the growth of the bone bases to

Address for correspondence:

Mathilde Tsang Tung

233, rue Sainte-Catherine - 33000 Bordeaux - France

E-mail: math_tsangtung@live.fr

Article received: 19-05-2017. Accepted for publication: 09-06-2017.

This is an Open Access article distributed under the terms of the Creative Commons Attribution License (http://creativecommons.org/licenses/by/4.0), which permits unrestricted use, distribution, and reproduction in any medium, provided the original work is properly cited. 
occlusion. The treatment of a tootharch disharmony is possible without extractions ${ }^{24}$.

Angle $26-27$, who was a great admirer of "Mother Nature, who created nothing in vain" emphasized the need to preserve all teeth. "Due to the masticatory function, Nature will stimulate development and straighten the roots of the teeth until they are in the correct position, but it takes a long time and requires a long and tedious period of contention." In addition, success depends on the bone growth, age, and general condition of the patient.

For this purpose, he introduced his "E-arch" 19 in 1912, which was designed to place the teeth on the ideal curve of the arch by creating a moderate transverse expansion and a vestibuloversion of the incisors. Little by little, this technique has had to compensate for the vagaries of natural evolution using more complicated mechanisms. Then came the Pin-and-Tube, which was difficult to manage as it was one of the first brackets, the Ribbonarch ${ }^{27}$ and finally in 1925, the Edgewise ${ }^{27}$, which is the basis of our current devices.

Tweed, a student of Angle, followed his teachings for years and found post-orthodontic treatment relapses in his patients treated with expansion. He changed his approach ${ }^{24}$. The skeletal base is determined genetically, so it became necessary to adapt the contents (teeth) to the container (dental arches), by performing alveolar compensations. Initially criticized, Tweed advocated using avulsions for the first four premolars, to adapt the dentition to the bone bases and achieve the goal of rigorous incisor repositioning according to the therapeutic design of the Tweed triangle $^{1}$ (1954). Its aim is to achieve treatment stability and a straight profile.

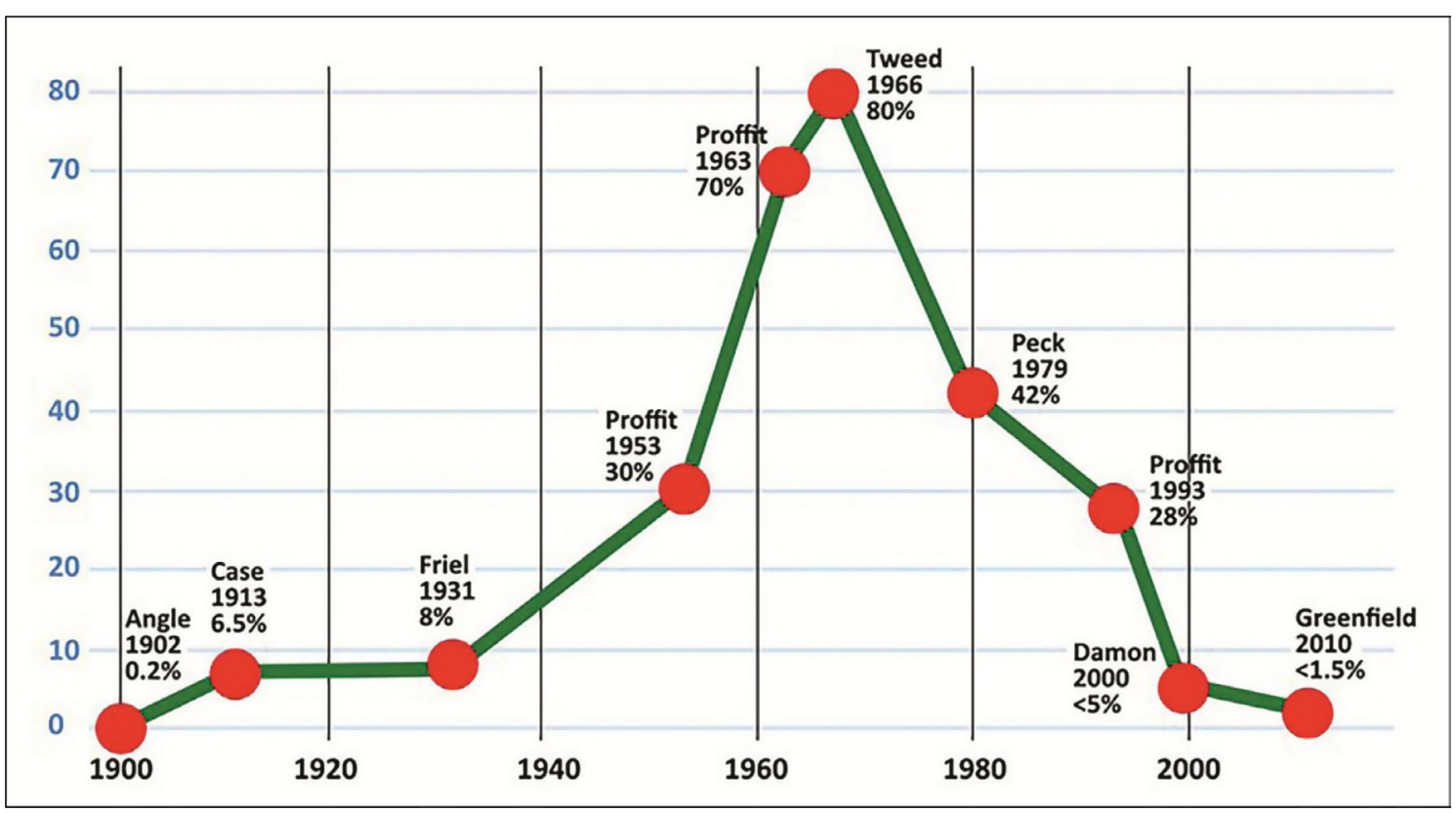

Figure 1

Peck Sheldon ${ }^{24}$. 
In the late 1950s, Ricketts ${ }^{9}$ developed the bioprogressive technique ${ }^{24}$, derived from the Edgewise, taking into account the skeletal base and the aesthetic and functional environment. Dual functional and mechanical disengagement ${ }^{9}$ acts according to the principle of the interaction between form and function. This orthodontic philosophy meets aesthetic, occlusal, periodontal, and neutralization objectives of the functional matrix. Distributed in Europe by Gugino ${ }^{9}$ in 1965, it was introduced to France by Philippe, Langlade, and Lejoyeux.

Damon $^{24}$ claimed that his methods could decrease the frequency of orthodontic extraction to $<5 \%$. And Green Eld $^{24}$ had supported its "coordinated arch development" that could decrease extractions for orthodontic treatment to $<1.5 \%$ of patient samples.

However, a big difference between the Damon and Angle approaches is that Damon uses resilient coppernickel-titanium (CuNiTi) alloys that distribute much softer expansion forces than Angle's wires which are made of gold, German silver, or chrome.

The evolution of treatment ideas is now more oriented toward treatments without extractions (Figure 1). Selfligating fasteners are the latest devices whose advocators, like Damon, claim a capacity to significantly decrease the indications of extractions and the duration of treatments.

\section{NEUROMUSCULAR PARADIGM}

\section{Mastication and ventilation}

Craniofacial growth depends on hereditary factors and orofacial functions, resulting in the facial structure, which supports ventilation, in particular, as well as mastication.

Form and function are the drivers of facial harmony. Taking into account the shape of the neuromuscular linguo-labio-jugal balance ${ }^{6,29}$ (Fig. 2) and functions, including ventilation, can lead to stable and harmonious treatments where expansion plays an active and effective role.

The growth of the bone bases is membranous in origin. It is a secondary and adaptive growth process in response to the demands of the environment. This is where "the template for the shape and function of the face" comes from. In the face of physiological muscular functions, growth will be normal. Conversely, growth will be disrupted if dysfunctions predominate.

The alveolar processes will play a catchup role between the mandibular and maxillary growth.

Masticatory forces direct the placement of teeth. The masticatory device will experience significant growth and multiple remodelings, which will alter its shape, position, and relationships.

The key to occlusion is the intercanine ratio, as it is this area that undergoes the fewest changes during morphogenesis. 


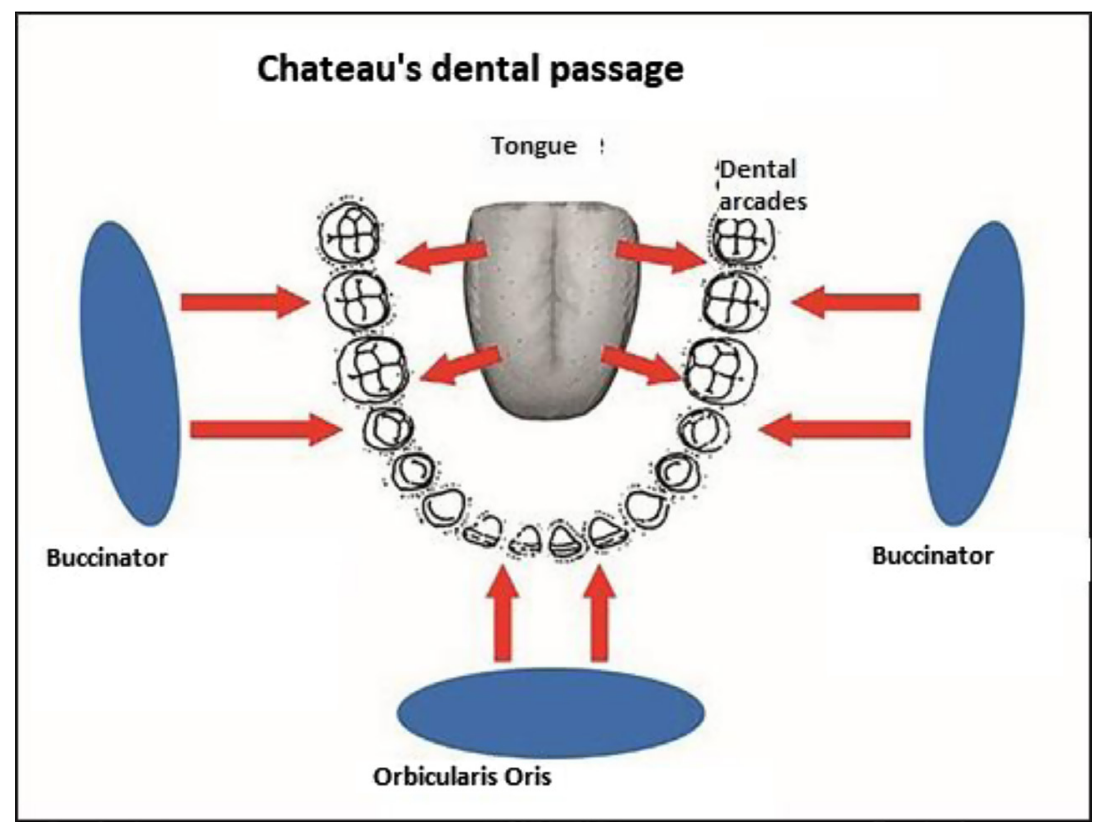

Figure 2

Château.

\section{Reduction of masticatory con- straints}

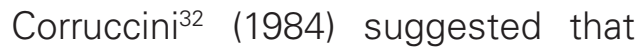
masticatory stress could be a regulating factor in jaw growth. Therefore, a reduction in functional stress would result in a smaller jaw size and increased occlusal variation.

Experiments in rats conducted by Kiliaridis ${ }^{4}$ indicated that changes in the function and structure of masticatory muscles, induced by a soft diet, affect both sutural growth and appositioning.

Varrela $^{32}$ (1992) performed a cephalometric comparison of two skull samples from different centuries, who were exposed to a hard or soft diet, to study the short-term variation of the craniofacial dimensions.
Hard food, which requires more strength and chewing time, promotes vertical ramus growth and anterior maxillary growth, as well as the anterior rotation of the mandible. This study supports the hypothesis that craniofacial skeletal growth is regulated by masticatory stress. It is suggested that dimensional changes and lack of tooth attrition may have contributed to the higher occlusal variation in modern individuals.

These changes seem to coincide with a cultural transition from hunter-gatherer lifestyle to agriculture. According to the authors, these adaptations are due to changing masticatory functional requirements (Carlson).

At present, the masticatory functional hypothesis seems to offer the strongest model by which many occlusal and craniofacial changes could be explained. 


\section{Consequences of these developments}

In the American school of thought led by Brodie and Tweed, the shape and size of the bone bases are genetically determined. The treatment can only act on the teeth themselves and serves to adapt the teeth to the size of the arches, which cannot be altered, hence the need for extractions and the interest in fixed multi-attachments.

In the European school of thought 6 , the bone bases are modeled by their functional role $^{29}$ that treatment can change, thus changing the course of growth. Establishing the link between etiologies and abnormalities makes it possible to develop methods of preventing and intercepting dysmorphoses.

\section{Modern man}

According to Planas ${ }^{28,}$, All the problems of our stomatognathic system, with rare exceptions, are caused by the masticatory functional impotence provoked by the insufficiency of the mechanical stresses engendered by our civilized diet." Since the middle of the Paleolithic era, human diet has changed considerably ${ }^{17,18}$ It is recognized that genetic and environmental factors influence facial growth, but it is also recognized today that diet and mastication contribute to facial variations affecting both size and shape ${ }^{16}$. Feeding behaviors ${ }^{17}$ produce progressive and continuous stimulations for the growth of the bone bases and alveolar processes.

\section{Physiopathology of mastication}

\section{Role on the facial structure}

The impact of masticatory function on maxillary growth was described by Planas ${ }^{28}$ in his "Laws of Development." The pressure transmitted to the bone by the teeth stimulates its growth and promotes the establishment of a balanced occlusion ${ }^{18}$.

The muscular stresses developed during the masticatory cycles stimulate the growth sites (midpalatal and periosteal sutures), resulting in the transverse growth of the bone bases and therefore in a transverse expansion of the dental arches. Physiologically, mastication consists of alternating unilateral chewing.

An experiment by Zink and Lieberman $^{33}$ conducted on men confirmed the link between the consistency of food and the muscular force developed. The consistency of the chewed food has a systematic impact on the maxillary alveolar transverse dimension (intermolar distance), with a harder diet generating more growth in this dimension. Food preparation and meat consumption (which provides more energy by chewing less) seem to be linked, in human evolution, with a decrease in facial volume, the tooth size, and the masticatory device, allowing a facial architecture adapted to other functions (for example phonation and language), apart from changes in the volume and shape of the brain.

\section{Causes and consequences of patho- logical mastication}

The rheological characteristics, i.e., food texture and hardness, will condition the masticatory cycles (Fig. 3). 


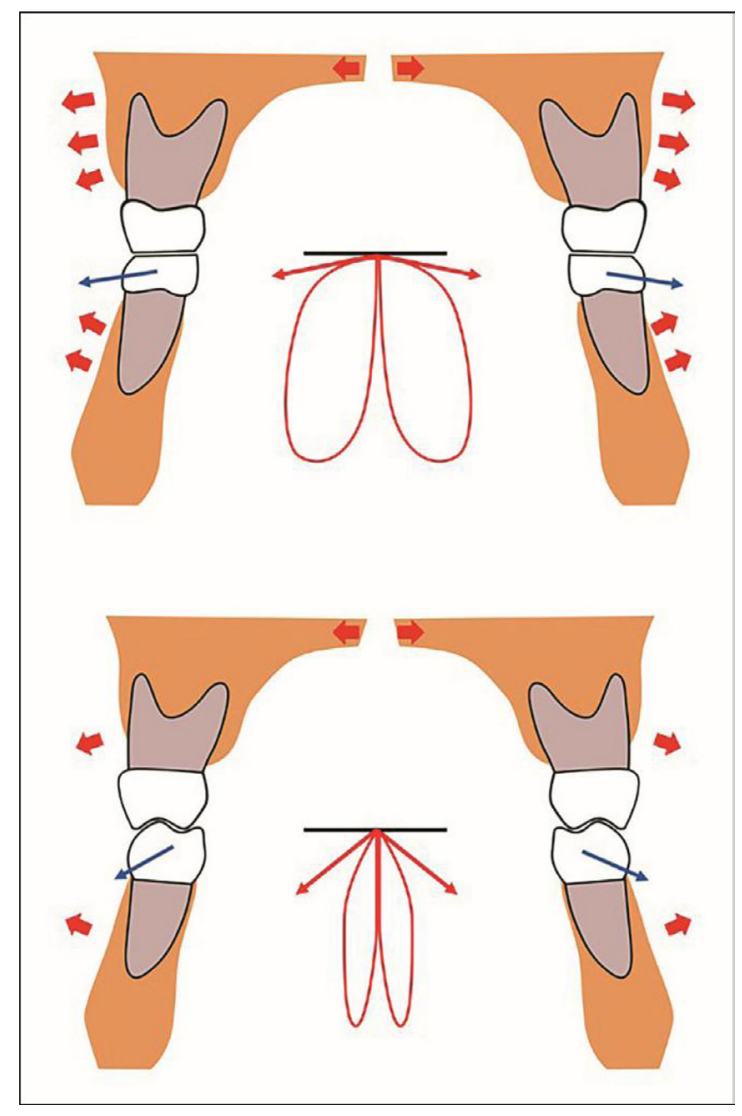

Figure 3

Alveolar solicitation and masticatory cycles during hard and soft chewing according to Limme ${ }^{17}$.

According to Peyron and Woda ${ }^{25}$, hard chewing leads to significant masticatory cycles with lateral and vertical movements that strain the sutures of the facial massif. Conversely, a soft and tender diet leads to shorter masticatory cycles, low muscular stress, absence of crushing, leading to nonattritional chewing and therefore an absence of mandibular lateral movements. The alteration of these cycles decreases the transverse dimension and is responsible for the appearance of dentoskeletal dysmorphias.

Engström's animal experiments ${ }^{8}$ showed the importance of masticatory function on sutural growth stimulation. The group receiving a hard diet shows a large interpremaxillary suture with many mitoses, indicative of suture activation and an active response mode. The group receiving a soft diet shows a narrow and fine suture, with few mitoses and a weak active suture already in the process of synostosis.

Ciochon et al. ${ }^{16}$ studied the influence of food consistency on mastication function and growth in miniature pigs. The masseter muscles and temporal muscles were more defined by $25 \%$ in pigs fed a hard diet compared to those fed a soft diet.

Kiliaridis ${ }^{12}$ showed that the reduction of muscular activity related to a softened diet modifies the structure of the masticatory muscles (size and type of fibers), which alters their contractile capacity. In animals, there is a decrease in bone apposition at the level of the insertion zones of the masticatory muscles and at the mandibular level.

Beecher et al. ${ }^{16}$ showed that an artificially tender diet was accompanied by a narrowness of the maxillae, gingival palate, malpositions, and dental congestion in monkeys.

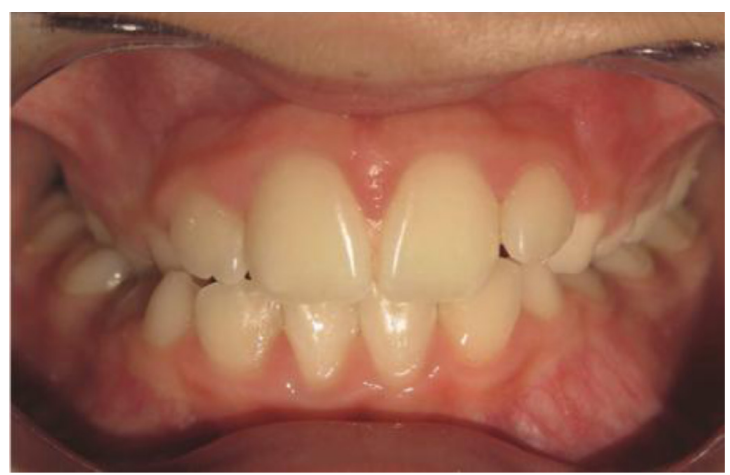

Figure 4

Maxillary endognathy with inverted right posterior occlusion (CHU patient). 
Andrade et al. ${ }^{30}$ suggested that the alteration of muscle function associated with the inverted posterior cruciate could decrease the intensity of the bite force, which is lower.

\section{PROGRESSION OF TRANSVERSE THERAPEUTICS}

\section{Maxillary expression in growing subjects}

Reverse unilateral posterior occlu$\operatorname{sion}^{30}$ is accompanied by functional and morphological asymmetries with an impact on the growth of mandibular bone structure, electromyographic (EMG) activity of masticatory muscles, and mastication cycle (Fig. 4).

The management of the transverse dimension must take into account a particular problem: When should it be dealt with? How often do relapses occur?

Ratios between an increase in the transverse dimension and changes in the perimeter and the width and length of the arch are used in the planning of orthodontic treatment and are often associated with the decision on extraction ${ }^{1,24}$.

\section{Disjunctor versus Quad Helix}

In 1841, Lefoulon introduced treatment without extraction and worked on expanding the transverse dimension of the maxilla. Orthopedic maxillary expansion treatments have been used for $>140$ years and have been developing since 1860. Described for the first time by Angle in a case report, after several controversies over its use, it was reintroduced in 1961 by Haas ${ }^{15}$. Rapid maxillary expansion (RME) ${ }^{15}$ has been the subject of numerous studies that have yielded valid and predictable results, justifying its widespread use. Since then, RME has been the treatment of choice in the treatment of maxillary endognathy (Fig. 5).

It is the most popular and most studied type of expansion in the international literature. Rodrigues notes more than 600 articles referenced in PubMed for the simple acronym RME.

Less predictably ${ }^{15}$, RME has also been linked to the improvement of respiratory disorders such as oral breathing and sleep apnea.

The maxillary expansion obtained after expansion (Fig. 6) is never purely orthopedic: there is 38\% skeletal expansion by action on the medial palatal suture and $13 \%$ alveolar expansion

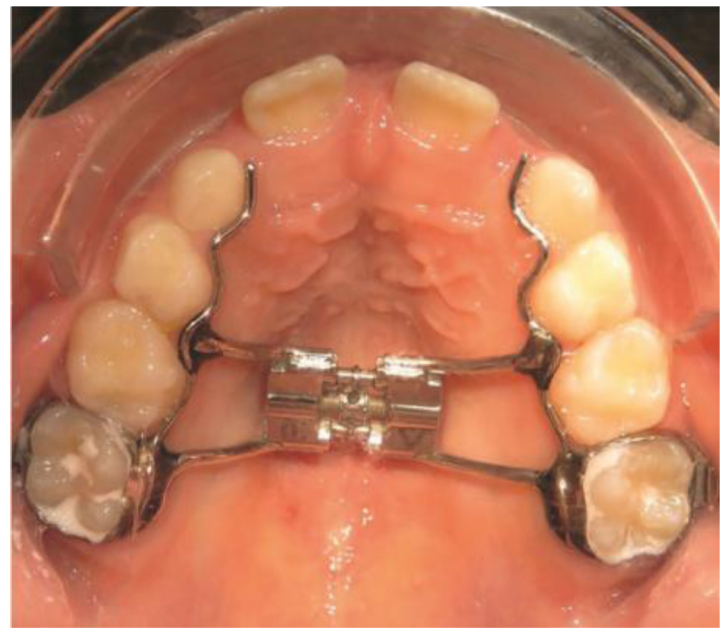

Figure 4

Disjunctor with dental support on rings (patient CHU). 


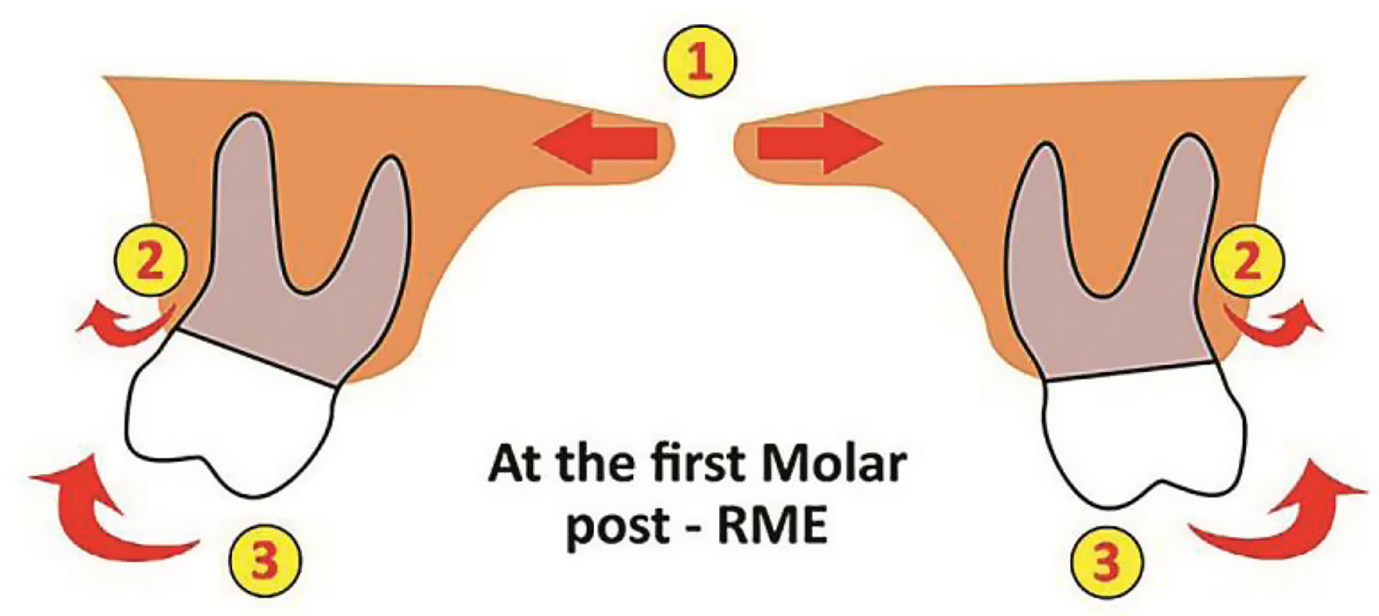

Figure 6

Effects of maxillary expansion according to Garrett ${ }^{15}$.

by vestibular apposition and palatal resorption, and $49 \%$ vestibuloversion of the teeth.

Comparison of anteroposterior cephalometry $^{15}$ in patients with palatal implants having maxillary expansion demonstrated that approximately 50\% of the expansion achieved by RME in children was skeletal and the remainder was dentoalveolar.

However, maxillary orthopedic expansion has its limits when the patient is older. The intermaxillary sutures ossify as soon as the growth of the maxilla stops, at the age of approximately 14 years for girls and 17 years for boys. In addition, the maxillary pillars appear to be major obstacles, significantly decreasing its success rate in adults by increasing the rate of recurrence and the appearance of unwanted maxillary movements, as well as numerous side effects: intense pain and edema upon activation of the disjunctor, alveolar dehiscence, fenestration and root resorption, gingival recessions, severe and irreversible periodontal problems related to a vestibular version of the teeth caused by the disjunctor rather than the desired movement of the bone bases.

Some orthodontists propose to decrease the speed of expansion to avoid side effects.

Slow maxillary expansion ${ }^{14}$ (Fig. 7) is recommended in prepubertal subjects where the application of weak forces

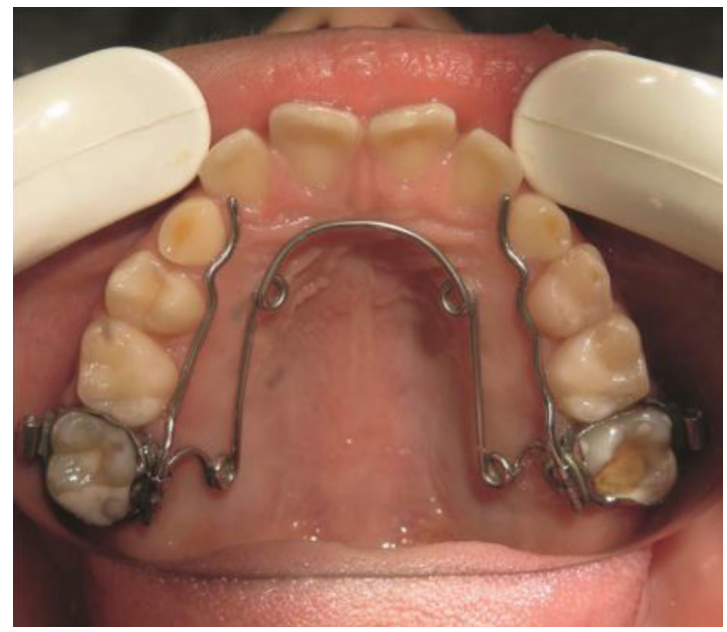

Figure 7

Quad Helix in a 7-year-old child (CHU patient). 
is sufficient with deciduous dentition or early mixed dentition. The use of stronger forces in young children under 7 years of age may cause deformation of facial structures, especially in the nasal area.

According to Gill ${ }^{14}$, slow expansion would produce less resistance in the perimaxillary structures and progressive mineralization, which will ensure the physiological integrity of the suture while decreasing the negative effects of heavy orthopedic forces. Some authors even consider this mode of expansion more stable because it allows a reossification and a vascular development concomitant to the sutural expansion.

Recent data from the literature reveal that these techniques are less reliable and should be reserved for mild endomaxillary issues in adults with a healthy periodontium because some degree of dental version is unavoidable. Surgical expansion is the technique of choice at the end of growth because it makes it possible to eliminate any bone resist-

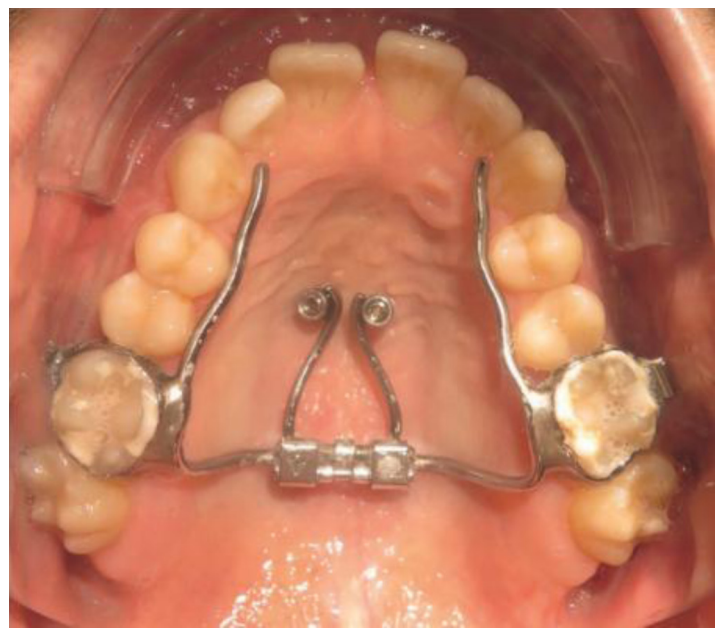

Figure 8

Maxillary distraction on miniscrews (CHU patient). ance to the lateral movements of one or both upper maxillae.

\section{Bone anchorage versus dental anchorage}

According to a review of the literature published in 2005 by Lagravère et al. ${ }^{14}$, only $25 \%$ of this expansion would be skeletal; hence, the majority of the expansion would be alveolar. On the other hand, Krebs, in 1964, attributed 50\% to skeletal expansion in children.

These changes in opinion lead us to look for a way to increase the ratio of skeletal effects to alveolar effects. Temporary skeletal anchoring was therefore presented as a true revolution in orthodontic biomechanics.

The invention of mini-implants ${ }^{5}$ over the last 20 years offers new treatment alternatives by allowing dental movements when conventional approaches are limited ${ }^{1}$. Their application makes it possible to counter the undesirable effects and to push the limits of the use of conventional devices (Figure 8).

Miniscrews are a real revolution in the management of orthodontic anchorage. This concept, which is not invasive and simple to implement must, however, follow a thoughtful and individualized biomechanical strategy.

Historically, the palatal suture was considered the main area of resistance. Isaacson et al. have explained that the total resistance offered by the bone structure of the maxillofacial complex becomes higher as the patient increases in age and thus becomes more mature at the skeletal level, and that the major sites of resistance come from the different junctions between the maxilla and the craniofacial complex.

In 1975 and 1976 respectively, Lines as well as Bell and Epker ${ }^{2}$ confirmed 
that the areas of greatest resistance were the frontomaxillary, zygomaticotemporal, and zygomaticomaxillary sutures. The identification of these resistance zones stimulated the development of the various EPRAC techniques. It has been proposed that an osteotomy of the lateral maxillary pillars should be performed as well as attempts to free the maxilla in the posterior region.

\section{Orthodontic development of the arches}

The development of the "pentamorphic" system from studies of untreated normal arches and the long-term stability of the treatments determines the best shape for the patient: the diagnostic arch form is obtained. An arch can be reformed either by taking the predefined diagnostic arcade or a treatment arcade shape chosen according to the treatment objectives.

In the Tweed technique ${ }^{1}$, the arch form must be in harmony with the general shape of its skeletal support in respect to the muscular environment to achieve a functional and stable occlusion.

\section{Muscle analysis after maxillary expansion}

De Rossi ${ }^{7}$ compared electromyographic the EMG activity of the masseter and the temporal muscles after maxillary expansion in two periods separated by an interval of 5 months: T1 (before treatment) and T2 (1 week after the removal of the device), according to three positions: rest (10 s), maximum tight occlusion with or without cotton (4 s), and normal mastication (10 s). The analysis showed that the activity of the muscles increased significantly after the removal of the device in all three positions. This can be explained by the fact that the occlusion has been balanced and that the musculature is trying to adapt to the new occlusal condition.

During arch expansion, the teeth move against the perioral soft tissues, which affects the balance between the labial and lingual forces acting on the teeth. A study was conducted to measure jugal pressure at the upper first molar in patients before and after expansion and then 3 months after contention. Oral pressure went from $3 \mathrm{~g} / \mathrm{cm}^{2}$ before expansion to $9 \mathrm{~g} / \mathrm{cm}^{2}$ after, an increase of $0.6 \mathrm{~g} / \mathrm{cm}^{2}$ for every millimeter of expansion.

However, after expansion, no soft tissue adaptation is observed with maintenance of this elevated zygomatic pressure which results in recurrence of treatment after the third month of stabilization. The initial assumption of a return to the initial value of the post-expansion pressure has not been proven.

D. Halazonetis et al. ${ }^{10}$ (1994) also concluded that after expansion, no soft tissue adaptation occurred, resulting in the maintenance of high jugal pressure leading to recurrence of the transverse direction at 3 months after contention.

In contrast, experimental evidence has shown that pressure on the oral surface of teeth increases immediately after expansion. Over time, these increased pressures gradually decrease toward pre-expansion values, indicating an adaptive response of the labial muscles to the new position of the teeth.

In a study by Küçükkeles et al. ${ }^{13}$ (2003), after recording the pressures observed on the tongue, lips, and 
cheeks before and after expansion and during the period of contention (at 1 , 2 , and 3 months), the physiological balance was regained.

The increased jugal pressure during the expansion returns to its preexpansion value after 3 months of contention. The lingual pressure, which decreased during expansion, increased after expansion but it did not return to pre-expansion values. On the one hand, the jugal pressure decreased and the lingual pressure increased. These values show that the lip and cheeks adapt more easily to the new position of the dental arches after 3 months while the tongue takes longer to adapt.

The muscles of the cheeks, tongue, and lips tend to return to their initial values at the end of 3 months of contention. Previous studies have shown that the forces produced by expansion disappear at the end of the sixth week and the suture remineralizes 3 months after expansion. The causes of longterm relapses need to be examined further.

\section{STABILITY OF EXPANSION}

\section{Recurrence and expansion}

Patients who relapse ${ }^{3}$ have been subjects of interest to orthodontists for more than a century, even though opinions have gradually changed.

At the end of $19^{\text {th }}$ century, Angle, Case, Kingsley, and Dewey thought

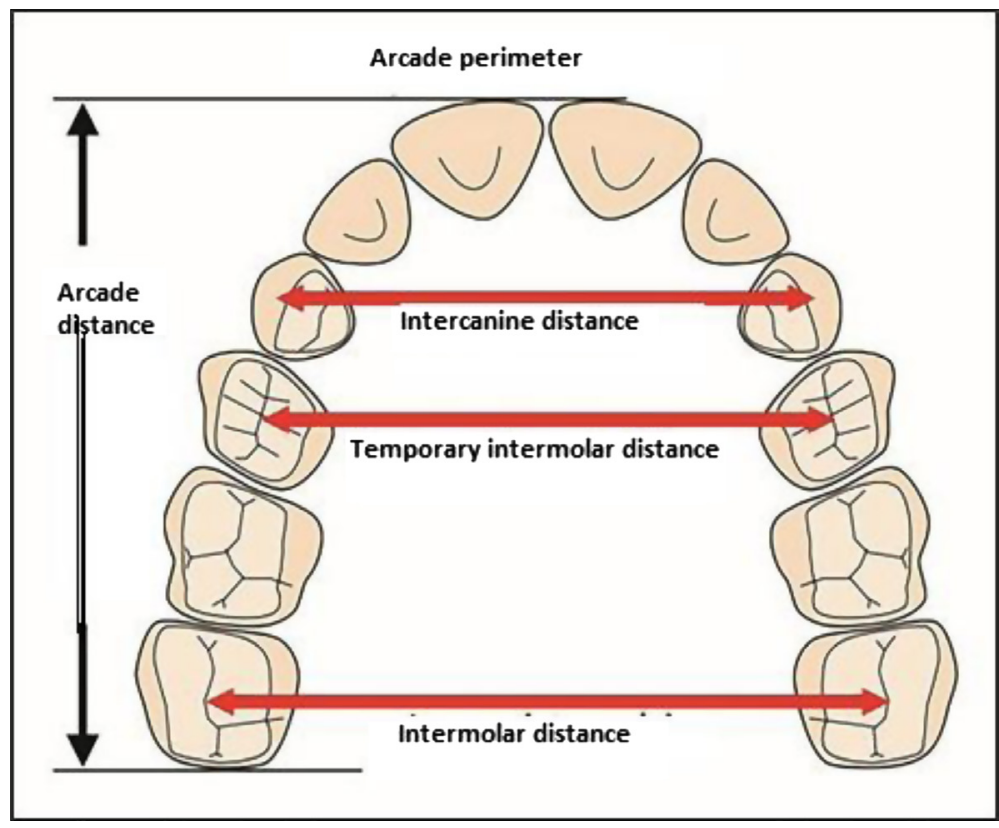

Figure 9

Dental arch according to Izard. 
that a good occlusion is the most important factor for stability.

Around 1920, Lundström, Mac Cauley and Strang gave great importance to intercanine and intermolar arches (Fig. 9).

For Tweed (1946) and Nance (1947), the mandibular teeth must have a good relationship with the basal bone. It is important not to increase the length of the arch and avoid excessive vestibular or lingual versions.

Dewey and Anderson have said that muscular pressures and balance are a very important natural retention force.

According to the Tweed Foundation?, respecting the limits of the teeth is essential to arrive at a functional and stable occlusion. In the sagittal dimension, the position of the anterior teeth is represented by the diagnosis triangle of Tweed. However, in the transverse dimension, the teeth are in a position of neuromuscular equilibrium represented by the initial position of the teeth, which must be respected at all costs.

Brodie, taking up the ideas of Rogers and Dewey, stresses the need to get a good muscle balance.

Changing the shape of the dental arches without modifying the functional roles which led to the problem will definitely result in a relapse ${ }^{3}$.

Oral ventilation induces a lack of transverse development of the nasal fossae and the maxilla (recurrence of endognathy or endoalveolia).

\section{Providing strong masticatory constraints}

Mastication is indispensable to the alveolar growth of the bone bases. In

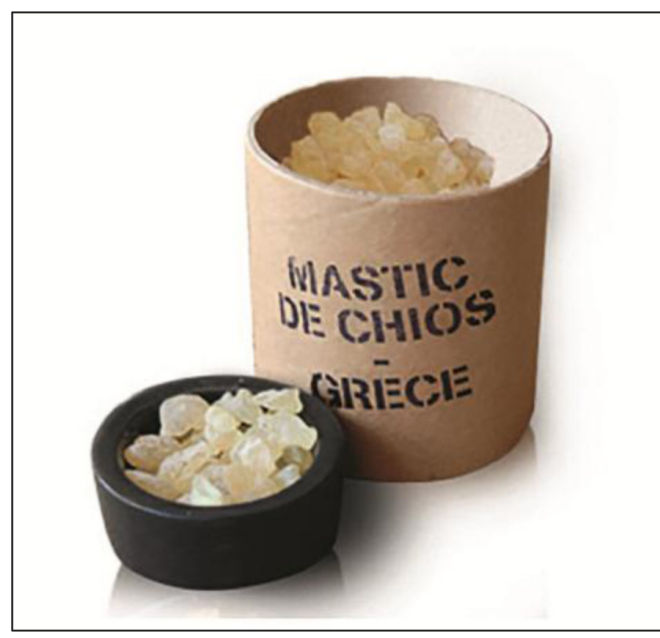

Figure 10

Chios gum.

our human society, dental evolution has led to the frequent appearance of essentially transverse malocclusions due to the absence of significant masticatory stress. Of course, we cannot change current human anatomy, nor change the diet but we must realize that the individual is in hypofunction and requires strong masticatory constraints to help stimulate proper occlusion while respecting the surrounding muscle balance.

\section{Chios gum}

Famous since ancient times, the natural chewing gum from Chios in Greece $^{19}$ is a pure semitransparent resin extracted from the tree Pistacia lentiscus chia, which only grows in the southern part of Chios (Fig. 10).

It is natural, and has antioxidant, anti-inflammatory, and antimicrobial properties and is used both for its unique aroma and healing properties. Mastication of natural resins is a very old human habit (at least 6,000 years old), according to some archaeological findings. 
This gum has two advantages ${ }^{19}$. First, its effectiveness has been proven. Second, its use does not lead to any ethical problem because it is a natural product, having received the European standard for over-the-counter use without requiring a prescription.

When chewed, the sap crystals break and form a hard gum with a very fresh, pine-flavored taste. This requires significant chewing efforts. It causes an increase in muscle tone and a better ability to close the mouth.

\section{Uses in previous studies}

In the literature, among the tools used to increase the masticatory capacities of the patient, gums have been prescribed in addition to orthodontic treatments. Various studies have shown the interest of gum-chewing exercises, usually hard gums, in the increase in occlusal strength, chewing efficiency, and occlusal quality. Kachi ${ }^{23}$ in 1987 showed that masticatory efficacy was increased by chewing a hard gum three times a day for 3 min for 13 weeks for children.

Ingervall et al. ${ }^{11,23}$, in 1987, stressed the change in the direction of growth in the vertical dimension in hyperdivergent subjects following gum-chewing exercises lasting $1 \mathrm{~h}$ per day.

Ono et al. ${ }^{11}$, in 1992, observed a significant increase in the maximum occlusal strength in young children after 3 months of chewing exercises with a high strength chewing gum designed to stimulate chewing activity. However, there was no control group in this study.

Kiliaridis et al. ${ }^{12}$, in 1995, reported that chewing a hard gum for $1 \mathrm{~h}$ a day for 28 days significantly increases maximum occlusal strength in adults.

Okazaki et al. ${ }^{22}$, in 1999, reported that chewing gum for 3 min a day for 1 year significantly increases masticatory efficiency and occlusal strength in young children.

Masumoto et al. ${ }^{20}$, in 2009, reported that daily chewing of gum before

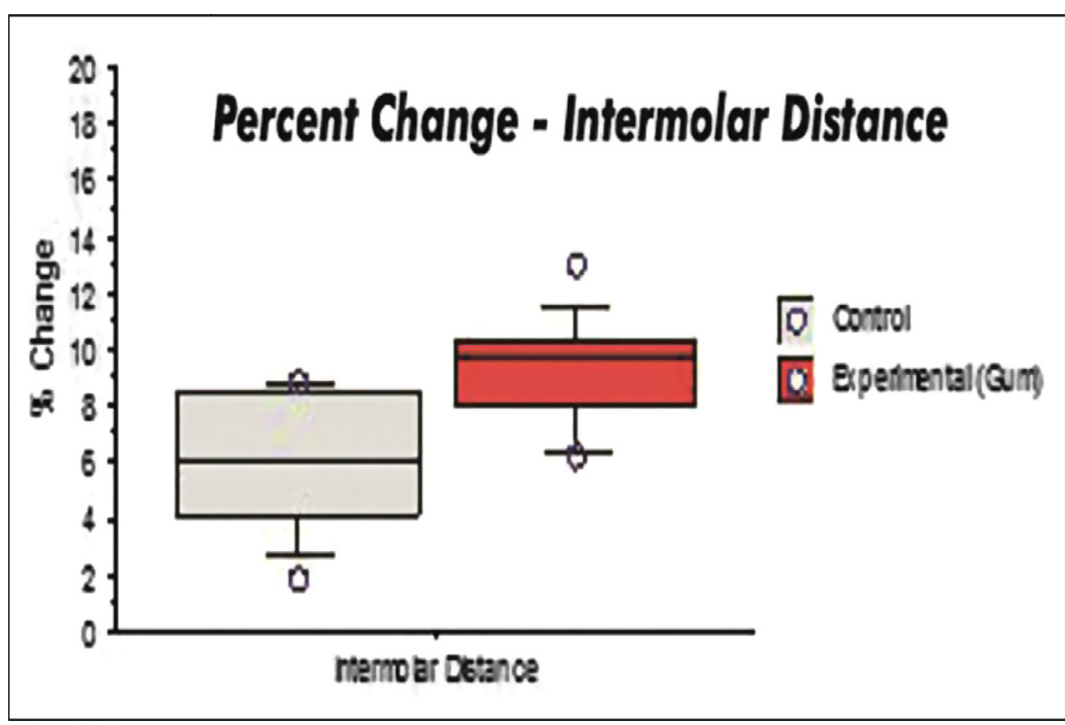

Figure 11

Increased stability of the intermolar dimension in subjects chewing gum. 
and after three meals for 4 weeks increases occlusal contact and occlusal strength in adults.

Ohira et al. ${ }^{21}$, in 2012, proved that the introduction of strong masticatory constraints significantly increases the activity of the masticatory musculature and its efficiency, as well as the occlusal force, thus improving the masticatory function. They studied, on the one hand, the maximum occlusal force via an occlusal force meter and, on the other hand, masticatory performance via chewing gum of interchangeable color: $3 \mathrm{~g}$ of a green-yellowish gum which could change color based on Xylitol ${ }^{\circledR}$ from Japan. (Lotte Co).

He et al. ${ }^{31}$, in 2013, reported that chewing a silicone gum for 4 months increased muscle strength and maximum occlusal strength, but after stopping the exercise they found a decrease in these values.

Makaremi and De Brondeau ${ }^{19}$, in 2015, showed that chewing gum for 6 months (Fig. 11), after transverse expansion by Quad Helix, improved the stability of the expansion (intermolar distance), and led to a stable occlusion, with straightening of the molar axes in the frontal plane (maintaining radiculovestibular torque).

\section{CONCLUSION}

The causes of relapses of the transverse dimension after mechanical maxillary expansion are multifactorial, involving functional and mechanical factors. These different growth factors explain the predominant influence of dysfunctions in the etiology of transverse anomalies and the necessary
Zink and Makaremi ${ }^{19}$ performed a comparative study of the range of mandibular movements during the chewing of Chios gum and a Hollywood chewing gum $®$ using three-dimensional motion sensors to understand the real effect of the gum on the masticatory musculature.

The exercises involved chewing $1.5 \mathrm{~g}$ of hard Chios gum and $1.5 \mathrm{~g}$ of regular chewing gum. EMG recordings measured masseter activity with a request for unilateral left-sided chewing during the experimental phase.

The results showed that chewing hard gum generates a significant increase in muscle activity. A 93\% increase in the activity of the left and the left masseter (mastication side) and an increase of $326 \%$ in the muscular activity of the right masseter. This difference is justified by the need to stabilize the nonmasticating side given the intensity of the occlusal pressure.

The contribution of strong masticatory constraints by the chewing of the Chios gum generates forces that stimulate growth and bone density. Strengthening this muscle tone will influence the direction of growth optimally.

reeducation of patients to maintain the stability of the treatment results.

The transverse correction of the occlusion and the maxillary dimensions contributes to the normalization of functions, which will resume their role of harmonious growth ensuring the stability of the result obtained. 
The evolution of our diet over the past centuries has resulted in mastication that has become almost "impotent": dietary habits (sugar, cooked dishes, drinks) and soft food consistency (refined and tender foods) have changed and resulted in a significant decrease in mastication. The low workload of this masticatory function deprives us of the functional stimulations for growth necessary for the physiological placement of the maxillary anatomy.

Neglecting dysfunctions would leave in place the mechanisms that generated the initial malocclusion, leading to a recurrence; hence, they must be corrected.

The prescription of hard gums, in our daily practice, after maxillary expansion would stimulate the growth of the alveolar processes in the child, as well as the maintenance of the transverse expansion and also the straightening of the axes of the molars.

Conflict of interest: The authors declare that they have no conflict of interest.

\section{BIBLIOGRAPHY}

1. Amm EW, Bou-Serhal JP. The arcade shape according to the Tweed-Merrifield philosophy: individualization and standardization test. Orthod en 2003;74:1-18.

2. Bell WH, Epker BN. Surgical-orthodontic expansion of the maxilla. Am J Orthod 1976;70 (5):517-528.

3. Blake M., Bibby K. Retention and stability: A review of the literature. Am J Orthod Dentofacial Orthop 1998;114(3):299-306.

4. Ares, Kiliaridis S. Dento-skeletal adaptation after bite-raising in growing rats with different masticatory muscle capacities. Eur J Orthod 2002;24(3):223-237.

5. Carlson C, Sung J, McComb RW, Machado AW, Moon W. Microimplant-assisted rapid palatal expansion appliance to orthopedically correct transverse maxillary deficiency in an adult. Am J Orthod Dentofacial Orthop 2016;149(5):716-728.

6. Cauhepe J, Fieux J, Coutand A, Bouvet JM. The morphogenetic role of neuromuscular behavior. Rev Stom 1955;56(7):535-546.

7. De Rossi M, Rossi A, Hallak JE, Vitti M, Regalo SC. Electromyographic assessment in children having rapid n. expansion. Am J Orthod Dentofacial Orthop 2009;136(3):355-360.

8. Engström C, Kiliaridis S, Thilander B. The relationship between masticatory function and craniofacial morphology. II. A histological study in the growing rat fed a soft diet. EUR J Orthod 1968;8)(4):271-279.

9. Gugino C, from J. The concept of unlocking: the interaction between form and function. Rev Orthop Dento Faciale 2000;34:83-108.

10. Halazonetis DJ, Katsavrias E, Spyropoulos MN. Changes in cheek pressure following rapid n. expansion. Eur J Orthod 1994;16(4):295-300.

11. Ingervall $B$, Bitsanis $E$. A pilot study of the effect of masticatory muscles on facial growth in longface children. EUR J Orthod 1987;9 (1):15-23. 
12. Kiliaridis S, Tzakis MG, Carlsson GE. Effects of fatigue and chewing training on maximum bite strength and endurance. Am J Orthod Dentofacial Orthop 1995;107(4):372-378.

13. Küçükkeles N, Ceylanoglu C. Changes in lip, cheek, tongue pressures after rapid maxillary expansion using a diaphragm pressure transducer. Orthod Angle 2003;73(6):662-668.

14. Lagravère MO, Major PW, Flores-Mir C. Skeletal and dental changes with fixed slow maxillary expansion treatment: a systematic review. J Am Dent Assoc 2005;136(2):194-199.

15. Lagravere MO, Heo G, Major PW, Flores-Mir C. Metaanalysis of immediate changes with rapid maxillary expansion treatment. J Am Dent Assoc 2006;137(1):4453.

16. Lieberman, Krovitz GE, Yates FW, Devlin M, St. Claire M. Effects of food processing on masticatory strain and craniofacial growth in a retrognathic face. J Hum Evol 2004;46(6): 655-677.

17. Limm M. Food ducts and growth of dental arches. Rev Orthop Dento Faciale 2002;36: 289-309.

18. Limm M. Food diversification and dental development: importance of eating habits of young children for the prevention of orthodontic dysmorphosis. 2010 Elsevier Masson SAS. Archives of Pediatrics 2010;17:S213-S219.

19. Makaremi M, Zink K, De Brondeau F. contribution of masticatrices constraints in the stabilization of maxillary expansion. Rev Orthop Dento Faciale 2015;49:11-20.

20. Masumoto N, Yamaguchi K, Fujimoto S. Daily chewing gum exercise for stabilizing the vertical occlusion. J Oral Rehabilitatio 2009;36(12):857-863.

21. Ohira A, Ono Y, Yano N, Takagi Y. The effect of chewing exercise in child preschool on maximum bite force and masticatory performance. Int J Online Tooth 2012;22(2):146-153.

22. Okazaki M, Takahashi K., Oka T. Effectiveness of nutrition education. J Child Health 1999; 58:575-586.

23. Parks LR, Buschang PH, Alexander RA, Deshow P, Rossouw PE. Masticatory exercise as an adjunctive treatment for hyperdivergent patients. Angle Orthod 2007;77(3):457-462.

24. Peck S. Extractions, retention and stability: the search for orthodontic truth. Eur J Orthod 2017;39(2):109115.

25. Peyron MA, Woda A. Adaptation of chewing on the mechanical properties of the food. Rev Orthop Dento facial 2001;35:405-420.

26. Phillipe J. Why, how, when was born the edgewise? Rev Orthop Dento Faciale 2008;42: 113-121.

27. Philippe J. History of behavioral therapy. Rev Orthop Dento Faciale 2012;46:111-117.

28. Planas P. Neuro-occlusal rehabilitation. Translation of Mr. Chateau, J. Kolf, J. Kolf. Paris, Masson.

29. Profitt WR. Equilibrium theory revisited. Angle Orthod 1978;48(3):175-187.

30. Raberin M. Pathologies and therapies of the cross-cutting dimension in mixed dentition. Consequences on the muscular balance. Orthod Fr 2001;72,1-2:131-142.

31. Stavropoulos D, Hagberg C, Hakeberg M, Mohlin B. Effects of masticatory muscle training on maximum bite strength and muscular endurance. ACTA Odontol Scand 2013;71(3-4): 863-869.

32. Varrela J. Dimensional variation of craniofacial structures in relation to changing masticatory-functional demands. Eur J Orthod 1992;14(1):31-36.

33. Zink KD, Lieberman DE. Impact of meat and lower palaeolithic food processing techniques on chewing in humans. Nature 2016;24;531(7595):500-503. 